CHAPTER 12

\title{
Preparing Manuscripts and Responding to Reviewers' Reports: Inside the Editorial Black Box
}

\author{
Ian Stolerman and Richard Pates
}

\section{Introduction}

This chapter describes how the peer-review process works and presents suggestions to authors of manuscripts. It is based on the experiences of scientists and clinicians who have many years of experience as editors of prominent addiction journals. The task of the editor is to publish manuscripts appropriate for the journal and to assist would-be authors in the production of suitable material. Many of the problems facing authors writing for scholarly peer-reviewed journals in the addiction field are similar to those in other fields. Therefore, it is recommended that readers consult one or more of the full-length books that have already been published in this general area. Hundreds of books have been published, as can be seen by searching Amazon.com or PubMed for "scientific writing." For example, a search of Amazon.com on December 1, 2014, produced 16,904 results, many of which were relevant. A short list of recent books is provided at the end of this chapter in Appendix A.

Nearly all academic journals now work exclusively with computerized systems that allow for submitting manuscripts, sending articles to reviewers, responding to the reviewers' comments, and making a decision on the manuscript (e.g., accept/minor changes/major changes/reject). The advantages of these systems are increased efficiency for the editorial staff and an easier submission role for the author. It also makes it easier to keep track of manuscripts. As a rule, nearly all the communications to and from the journal are now done electronically.

\section{How to cite this book chapter:}

Stolerman, I and Pates, R. 2017. Preparing Manuscripts and Responding to Reviewers'

Reports: Inside the Editorial Black Box. In: Babor, T F, Stenius, K, Pates, R,

Miovský, M, O’Reilly, J and Candon, P. (eds.) Publishing Addiction Science: A

Guide for the Perplexed, Pp. 229-244. London: Ubiquity Press. DOI: https://doi. org/10.5334/bbd.l. License: CC-BY 4.0. 


\section{Triage: the First Selection}

The author's quest to find a suitable publication outlet ends with a letter stating, "I am pleased to inform you that your manuscript is acceptable for publication...." But the first step is to get the manuscript into the peer-review system. Yet having your article peer reviewed is not the inevitable consequence of submitting to a peer-reviewed journal. Some journals state formally that they operate a system of "triage," whereby the editor or his or her assistants decide which submitted articles will be entered into the peer-review process. In practice, it is likely that all journals have such a system to protect the profile of the journal and to avoid bothering authors and peer reviewers with a long and laborious evaluation process when it is easy to predict a negative result (see Box 12.1). Thus, if something is received that clearly has no hope of acceptance, it may be rejected without review. Here, the difference between journals is quantitative rather than qualitative: In the journals of highest impact in science and medicine generally, including addiction research, it may be that more than half of the submissions are rejected at this stage. Some addiction journals will, however, accept almost all articles, or reject only $20 \%$ or $25 \%$. For some information on acceptance rates of addiction journals, see Chapter 3 and its appendix.

There are some aspects of manuscript preparation that are so easy that everyone should get them right. To ensure your manuscript has the best chance of passing through triage, make sure you do all these things as set out in the instructions to authors that every journal provides. Follow all advice and recommendations exactly, format your submission precisely as requested, make sure that all sections are complete, and be sure that no tables, figures, or figure legends are missing. Check the reference list to ensure that all cited references are in it, and no others. Check the accuracy of each citation. Look in the journal to see exactly how references are styled. Then check them over again, after you have made the corrections, until no more errors can be found. This sort of work is tedious but does not need expensive resources, profound knowledge of the subject, or outstanding intellectual ability. If the editor sees at a glance that you do not even get these straightforward, mechanical things right, he or she may well develop a jaundiced view about your capability to deal with more complex matters. Try to look at your own manuscript as an editor might. If you do not bother to do the easier things required of an author, the editor might reasonably conclude that you will not be able to do complex revisions either, and you may not be given the opportunity to revise and resubmit.

\section{Communication with more Experienced Writers}

Would-be authors may seek the advice of more experienced colleagues at almost any stage of the publication process. When planning a publication, discussion 
- The submission is outside the scope of the journal (e.g., it is about a misused substance but it is not relevant in any discernible way to misuse of or dependence on it).

- The manuscript type is not appropriate (e.g., a case report is submitted to a journal that does not publish case reports).

- It contains clear ethical problems such as apparent violation of current generally accepted standards for the treatment of human or animal subjects.

- The article is poorly organized.

- The report is purely descriptive, has no hypotheses, or reaches no conclusions.

- There are major methodological weaknesses.

- The article appears to offer nothing new.

- Instructions to authors are flagrantly ignored in some way not mentioned here.

Box 12.1: Reasons for rejection by triage.

Note: The editor has a duty to reviewers, as well as to authors, and tries not to waste reviewers' time by requesting evaluations of work that has no chance of acceptance for one or more of the reasons above.

with colleagues after presenting the work at a seminar in the home institution may yield some tips as to the type of journal that may be interested in the study. Subsequently, during preparation of the manuscript, it may be appropriate to seek the advice of local colleagues on technical aspects, such as statistical analyses. When a manuscript exists in a complete form, it is often immensely helpful to ask at least one person to read it and make comments and suggestions. People are very often willing to help if authors make clear that they value an expert opinion on aspects such as coverage of the literature, clarity, style, language, and validity of conclusions. If there is no person in the author's own institution, it is possible to approach outsiders and ask if they would be willing to comment. Both people whom you know personally and others who have published in the area are likely to feel flattered and pleased that you value their opinion and may well provide advice. The manuscript that cannot be improved has yet to be written, and even experienced authors often seek the opinions of colleagues because, after working on a manuscript for months through revision after revision, authors may find it difficult to spot the little problems that spring to the attention of a new reader. 


\section{Writing in a Foreign Language}

It is an unavoidable fact that many authors have to write in a language other than their own, and conveying complex scientific ideas with clarity and precision can be a difficult task even in one's native language. Authors may therefore find it worthwhile to seek the assistance of colleagues with more experience in writing in the chosen language and, if possible, enlist a native speaker of the language to correct the manuscript. If that is not possible, it may be necessary to obtain the assistance of a professional translator to suggest corrections. Journals provide varying amounts of assistance in the correction of errors after accepting a manuscript for publication, but they cannot do anything to assist reviewers of poorly written manuscripts. A fuller consideration of language issues and language editing services may be found in Chapter 4 .

\section{The Peer-Review Process: Selection of Reviewers}

The next step for the editor is selection of reviewers who will advise him or her of the strengths and weaknesses of the work and recommend whether or not it should be accepted, reconsidered after revision, or rejected outright. To improve the manuscript, reviewers are also expected to make constructive suggestions in a report that can be sent to the authors. The criteria used for selecting reviewers are diverse, and probably few if any journals have tightly defined procedures. Box 12.2 shows the main criteria used by editors to identify reviewers. The number of reviewers for each article varies within and between journals, but most commonly there are two. The editors of some journals may work with only one reviewer, but this seems to be increasingly rare. Occasionally, three or more reviewers are used, depending on the journal and the editor's perception of the complexity and significance of the work. For example, multidisciplinary manuscripts may require more than two reviewers to ensure sufficient expertise. Similarly, if a study seems likely to have a major practical impact, for example on policy or treatment, the editor may wish to be especially certain that it is assessed thoroughly. If the two reviewers initially selected disagree about the article, an editor may seek additional advice from a third person to reach a decision.

For all reports, regardless of whether they are quantitative or qualitative, each journal has its own set of instructions for reviewers; journals differ with respect to the attributes of their "ideal" manuscripts. There will sometimes be a requirement for reviewers to complete a questionnaire as part of the review, with ratings of the manuscript according to criteria such as importance and likely impact on the field, as well as technical competence. The reviewers are usually also asked to make a recommendation on the fate of the manuscript and to justify it in confidential comments to the editor. Finally, reviewers are in all cases expected to produce a report that the editor will forward to the 
authors. The main purposes of this report are (a) to make suggestions enabling the author to improve the manuscript and (b) to list criticisms that the reviewer believes need to be addressed if the report is to be published. The report to the authors should not include specific recommendations for acceptance or rejection of the manuscript because that decision is the editor's. Chapter 13 provides further advice on how to become a competent reviewer.

Reviewers are asked to act according to ethical guidelines that are presented and discussed elsewhere in this book (see Chapters 14 and 15). The task of the editor is to reconcile sometimes conflicting reports from different reviewers and to make a personal judgment based on a variety of other considerations. The task is made more difficult if the reviews contain conflicting recommendations for publication.

- Recognized expertise in the specific field of the manuscript as noted in the journal's database of previous reviewers and authors.

- Previous invitations to the reviewer that have resulted in thorough, well-written, polite reviews submitted in a timely manner.

- Record of recent publications in the field as determined by searches of databases such as MEDLINE and PsycINFO.

The following individuals are typically excluded from consideration as reviewers:

- Persons who are known to have a very close connection to the authors or to have a conflict of interest with the authors will be avoided.

- People who are currently reviewing another manuscript for the same journal or who have reviewed one within a set period (e.g., three months) will be avoided.

- Those who work is excessively praised or criticized in the manuscript to be assessed are avoided.

Box 12.2: Some criteria editors use to identify reviewers for a particular manuscript.

Note: Some journals ask authors to suggest reviewers or to name persons they do not wish to have as reviewers. How to use these suggestions is the editor's decision. Different bulleted points from those above will be used in combination to reach a decision on whom to invite, and there will inevitably be appreciable variations between journals with respect to the use of these different methods of selection. 


\section{Criteria for Evaluation of Manuscripts}

If the journal has published its instructions to reviewers or put them on a website, these instructions will give you an idea about the features at which both editors and reviewers will look. Many journals probably look for the same desirable features of highly rated studies.

If a study is quantitative, the criteria include the use of a sufficiently large and suitably representative sample of the population under study, the presence of a high response rate among invited participants, the use of valid measures, the absence of procedural biases, minimal confounding of one independent variable with another, and the use of appropriate controls. Similarly, reviewers will look for as full a description of the methods as available space allows, with reference to earlier publications that provide more detail and establish the validity of the methods and measuring instruments (where applicable). Results must be described in a clear and logical sequence, with all necessary information presented. No more detail should be provided than can be covered in the discussion section. The discussion should bring out the importance of all the main findings and indicate how the work advances the state of knowledge and understanding in the relevant subfield. In addition, alternative interpretations of the data may be given, thus acknowledging limitations of the study. Reviewers pay attention to all the preceding points-and to many others.

In quantitative research, the data analysis section is prone to several problems. These include the following:

- failing to deal adequately with confounding variables;

- claiming to have shown something without performing a (statistical) test that supports it directly and unequivocally;

- failing to control for multiple comparisons; and

- drawing inappropriate conclusions from non-significant associations or differences: we probably all realize that lack of significance means only that we have failed to find an effect and does not prove that no effect exists, but we don't always remember this in our enthusiasm to explain how our results fail to support the ideas of a scientist whose theory we dislike.

Authors developing reports of randomized controlled trials may wish to follow the CONSORT (Consolidated Standards of Reporting Trials) checklist, which includes 22 items considered essential to judge the reliability or relevance of the findings (presented in Appendix B to this chapter in slightly abbreviated form).

The criteria for the evaluation of qualitative reports vary depending on the type of data and methods of analysis (e.g., participant observation and ethnography, qualitative interviews, content analysis, textual analysis, discourse analysis, ethnography and conversation analysis). Chapter 8 provides more information about how to write and publish articles using qualitative methods. Most types of qualitative reports should do the following. 
- Give clear criteria for the selection of data or subjects. Position the material carefully in the social and cultural space. For example, different genres of fiction represent different segments of the culture.

- Present a detailed account of where and when data were collected or which existing data sets were used. In studies based on fieldwork, describe the relation between the fieldworkers and the subjects and discuss the possible influence of the data collection on the phenomenon under study. Keep careful records of the data so that they can be provided for independent examination if necessary.

- Clearly state how the analysis was done, with an indication of whether reliability was assessed-for instance by replicating the analysis.

- Describe any themes, concepts, and categories derived from the data. Divide the interpretation process into short steps, specifying rules of classification and interpretation.

- Outline steps taken to guard against selectivity in the use of data; discuss exceptions and deviant cases. Ideally, the reader should be able to apply the same classifications, take the same analytic steps, and reach the same kind of results with another data set.

- Present data systematically so that quotations, field notes, and so on are easily identifiable.

- Offer enough primary evidence to show a relation between evidence and conclusions, but avoid the presentation of too many illustrations; the focus should be on the most representative examples.

\section{Common Problems with Manuscripts}

All parts of a manuscript are open to criticism from the title onward. The first requirement for gaining the confidence of an editor or a reviewer is to describe the findings objectively and in a sober style without the use of hyperbolic language. If your data are good, they will speak for themselves. It is always better for the reader to find that the results themselves are stronger than you claim.

Every "data not shown" statement may raise reviewers' suspicions that the authors are trying to hide something. If there really is not enough space to show important data graphically or in tabular form, then give some examples of the more important of such results in the text (with means, standard errors, or other indicators of variance and numbers of subjects, if it is a quantitative study).

The discussion section is the most difficult part of a manuscript to write, and it often shows. Sometimes the opening paragraph is only a summary of the results, which is not satisfactory. One approach is to decide which are the main new findings, mention only them, and summarize two or three important conclusions that follow from them. It is also common to find that the discussion does not focus on the aims as stated in the introduction and sometimes 
discusses issues on which no background was given. Such a failure to place findings in the context of previous knowledge means that the case for publication is not made. Reviewers and editors want to know what is new, what is confirmatory, and what fails to confirm previous findings. Instead, authors may attempt to extract too much from their data by trying to address too many different issues. The effect of this error is to dilute strong conclusions with weakly supported ones, giving an overall unfavorable impression leading to rejection.

The discussion should also consider alternative interpretations of the study and acknowledge major limitations. These may arise from methodological weaknesses or unexpected findings that could not be pursued to a firm conclusion because of practical limitations, such as the project period coming to an end or a financial constraint (these nonscientific reasons do not need to be stated). If the reviewers discover these weaknesses, they will consider themselves smart and are likely to make sure you know it; if you show that you are aware of the limitations and understand the implications, they will perceive you as smart and honest, which counts for a lot.

Do not waste time and space discussing "trends" that are not statistically significant; if the effect is not there, its implications do not need discussing. Remember that there are more than enough "significant" effects that do not replicate and there is no need to create new myths. If you believe that a real and important difference was undetected because of a lack of statistical power, the study needs to be repeated; that may be a factor to discuss.

Somewhat different problems are associated with reviews and theoretical articles. If a review claims to be comprehensive, it should state the way the literature search was carried out and define the criteria used for including articles (see Chapter 9). Articles that do not claim to be integrative reviews but rather argue the case for a particular theoretical viewpoint or set of ideas are often less comprehensive. In such instances, authors often cite publications that support their own position in a rather uncritical manner, and they may refer to few or no articles that oppose it. Editors may then firmly but politely ask the author to state the assumptions made and ensure that the article clearly indicates any controversial issues. Alternatively, where the intention is to let a distinguished writer express a personal view based on his or her selective citation of the literature, it should be made clear that a case is being made for a theory and that a balanced assessment of the state of the field is not being attempted.

Finally, remember you are writing a scholarly article and not running a campaign! Do not enter into politics and polemics. For example, if your main finding is that a widely used intervention is less favorable than another that lacks some sort of official approval for general use in your country, make the case for its relative merits and, if appropriate, argue for a policy change. But do not abuse the politicians and do not keep repeating the argument in more and more florid and emphatic language. Political battles are not won in the pages of academic journals. 


\section{The Editor's Decision}

The much-anticipated response from the editor finally arrives, together with the statements from the reviewers. The editor will often need to reach a decision based on the balance between innovation and quality of work. The perfect manuscript would have important new ideas with far-reaching importance backed up by sound data obtained by means of thoroughly validated methods. In reality, such manuscripts are seen only rarely, if ever, and the editor and the reviewers have to make judgments. If the approach to a problem is highly novel or the study is a potential stimulus for further valuable work, a manuscript may be accepted with data that are less than wholly convincing. On the other hand, if there is not very much that is really new but the study is the first one to address a particular methodological weakness of previous work, then clear data of high quality will probably be essential.

The reviewers' reports and recommendations inevitably influence the editor's decision, but they are not the sole determining factors. Editors may study a manuscript in varying amounts of detail and may have concerns that are not reflected in reviewers' reports. These concerns may relate to any of the range of issues that the reviewers also address but may especially relate to the appropriateness of the subject matter for the journal, whether there are any ethical problems, and whether the importance of the work is sufficient to justify publication in their journal rather than in a publication of lesser status that may be struggling to fill its pages. Studies may be technically competent and presented well but may be unimportant because they merely confirm well-known facts or focus on apparently trivial issues. When the reports of reviewers are in agreement with each other, the editor will most frequently accept the recommendations made. It is a brave editor indeed who overturns the opinions of two independent experts-reviewers may soon stop assisting an editor who consistently ignores the advice given. When reviewers disagree, the editor may seek to sort out the matter by studying the manuscript and coming down in support of one or other reviewer; this is the ideal method if the editor can reach a clear view because he or she can reach a decision quickly without wasting another expert's valuable time. However, sometimes reviewers reach opposing conclusions on the basis of equally well-argued cases, and then the editor may feel it is essential to obtain advice from a third person. This is especially likely to occur if the work is outside the editor's main area of expertise.

When a third reviewer reaches a definite view supporting one or the other of the earlier reviewers, then the way forward is clear; but this does not always happen. If the first reviewer supports publication strongly and the second reviewer recommends rejection, the third reviewer quite often says the manuscript is weak but may reach publication standard after major revision; in such cases the contribution of the third reviewer may swing the decision one way or the other depending on the journal's needs at the time. If the journal is trying to 
raise the standard of published items, such marginal manuscripts will probably be rejected, whereas if the study is in a field that is under-represented in the journal, the editor may wish to include it. An editor may also seek to publish the article in a shorter form that reflects its lesser merits.

The abusive reviewer is a particular annoyance to editors. The most commonly identified, although happily quite rare, form of abuse occurs when a reviewer attempts a review of the author instead of the manuscript. It is one thing, and perfectly acceptable, to state that an argument is constructed poorly and is unconvincing, that it is presented badly, or that it does not take account of previous knowledge; it is quite another thing to assert that the author is stupid, careless, or ignorant. Editors have a duty to alter or remove such inappropriate remarks from a reviewer's report so that unnecessary distress is not caused and the author will be encouraged to improve the manuscript. If the reviewer is young and inexperienced, the editor may also explain the problem with the report, whereas a senior person will more likely not be invited to review again. Additional advice for inexperienced reviewers may be found in Chapter 13 of this book.

A particularly difficult situation arises if the review process generates suspicion that the author has engaged in scientific misconduct or another form of unethical behavior. Such misconduct may be either minor or major in nature, and the editor typically has available a range of sanctions to apply. These may include refusing to consider further work from the author, reporting the matter to the author's institution or employer, and publishing a statement in the journal to alert the scientific community to the issue. The availability of a code of practice by which editors can abide in such circumstances is very helpful (see The Farmington Consensus for the ethical practice guidelines developed by the International Society of Addiction Journal Editors). Editors are also wary of trying to resolve contentious ethical issues; they often do not have the resources to conduct a full investigation. Equally important, they cannot simply brush the matter aside by refusing to publish suspect material but must take reasonable steps to ensure that appropriate action is taken. These and other related issues are discussed in greater detail in Chapters 14 and 15, which deal with ethical considerations in scientific publishing.

\section{Responding to Reviewers' Reports: General Rules of Conduct}

Authors who regularly achieve immediate acceptance of a manuscript as submitted are rare indeed. Revisions are almost always required before acceptance, and in many cases a final decision cannot be reached until the revised version has been assessed. Therefore, the way in which authors respond to the reports of reviewers and to the editor can have a major influence on the outcome. If editors invite resubmission, they expect to receive the manuscript back again. 
An invitation to resubmit is not a half-hearted and cowardly way of saying the work is unpublishable but rather an implicit suggestion that the editor remains interested in the article and that it is likely to be accepted if the author is responsive to the questions and recommendations of the reviewers. In such cases, it is nearly always worth resubmitting unless there is some clear and unavoidable requirement with which you cannot possibly comply. The remainder of this section offers guidance for authors on how to navigate through this maze successfully.

The overriding aim of the response is to engender trust among editors and reviewers. Authors should never claim to have made changes that in fact they have not done. If the cover letter says all requested changes have been made and an editor or reviewer checks two or three points at random and finds nothing much has changed, he or she may reject the manuscript without looking carefully at the rest of it. If you have made major changes by rewriting whole sections of the manuscript, state that is the case and identify the sections. Alternatively, if just a few words needed to be inserted or deleted, make clear which words were changed so that reviewers can see what has been done. If you were asked to shorten something, you should almost always do so and perhaps state by how much (i.e., by how many words or pages). Do not try to fool the editor by printing the new version in smaller type or by other stylistic changes. Be polite, even if you feel that the reviewers have not understood your intentions. When you have been through all the points of criticism, you should have an idea of the changes you think are appropriate. Will they be enough?

If after reading the reports you have concluded that none of the recommendations is worth accepting and you do not want to make any changes, it is common sense to take a break from the job and look at it again on another day! It is simply not realistic to expect editors and reviewers to accept that none of the changes they request and the criticisms they make is well founded. Reviewers spend anything from an hour to a full day preparing their reports. If you dismiss this effort out of hand, you will get nothing published. You must therefore aim to make changes to deal with as many as possible of the points raised and, preferably, with a clear majority of them.

Occasionally authors may feel that an editor's decision to reject their manuscript was unnecessary because the criticisms made could be answered through revisions. In such cases, in which there was no other clear reason given for rejection, authors may wish to seek approval to resubmit. For example, there may be no criticism of the conduct of the study or analysis of the data, but the reviewer may feel that the interpretation is so seriously flawed that the conclusions are not supported by the data. The manuscript might therefore be publishable if the authors are willing to revise their conclusions. Resubmission after rejection should be preceded by a carefully considered letter to the editor explaining why you believe that you can deal with the criticisms made. The editor will then decide whether to alter the previous negative decision and may agree to consider a revised version. Seek approval before resubmitting because 
if a rejected manuscript is resubmitted without prior agreement, it is very likely that the editor will refuse to consider it. Some journals have stated their appeals procedure whereas others deal with appeals on an ad hoc basis.

\section{The Cover Letter: Make Life Easier for the Editor}

Once you complete the changes to the manuscript, write a detailed reply to the reviewers. It is worth spending a significant amount of time getting your reply to reviewers as near to perfect as you can. Sometimes constructing the letter takes as long as revising the article, but it will not take as long as botching the job and then being obliged to reformat the manuscript for another journal to start the whole process over again. Nevertheless, it is best to keep this reply as short as possible. Typical successful replies will be in the range of one to three single-spaced pages. If the reviewer makes a point in just three lines and you need a page to rebut it, it is likely you have not gotten straight to the heart of the matter, and your reply will probably not be convincing. It is best to write the minimum needed to refute the criticism.

If the reviewer cannot understand a point that you made in the manuscript, it may be because he or she is lacking in intellectual capacity (as we often think when we encounter such comments on our own work). However, if one person does not follow what you have written, the same may apply to others. Reviewers are all published research workers and are often the very people whom you might hope would read your article; if a reviewer cannot understand your point, try to analyze your text to see how the misunderstanding may have arisen. Then make changes to ensure it will not happen again.

Do remember that if a reviewer asks a question, other readers may want to know the answer to it too. The answer should therefore usually be contained in the revised manuscript and not in the cover letter. Save the reviewers' time and they will love you; do not answer a question in the letter and then refer reviewers to a section in the manuscript that they have to read over and over to check if it is really there!

If possible, reply in numbered sections that correspond with the reviewers' numbered points. Explain the revisions you made to deal with most of the criticisms, and also explain why you did not deal with the rest. Describe briefly each change you made, referring to the relevant page or paragraph in the revised manuscript. Try not to respond in a combative, overly assertive style. If there are major and important changes recommended that you are sure are wrong, then present a concise, logically argued rebuttal. If there are minor changes requested that you feel do not really improve matters, do them anyway because it helps a lot if you can truthfully claim to have dealt with the majority of points. At all stages, remember that although reviewers and editors may appear to be distant, self-opinionated, and arrogant, they are also human beings with their 
own feelings, emotions, and problems. If you want acceptances, make life easy for them by writing clearly, and do not antagonize them with criticisms or gratuitous insults, however unwise and misguided you think the reviewers may be. It is also worth making the changes via a tracking system in a different color (such as red) on the manuscript to show clearly where changes, additions, and deletions have been made.

It is sensible to maximize and stress the points that you agree with that the reviewers wrote and to acknowledge their contribution when they have made suggestions that improve the manuscript. Do not build minor disagreements into major issues. You probably need to make only minor changes to accommodate them and then mention the changes in the cover letter and should not waste time arguing and or risk offending the reviewer in the process. However, it is not necessary or appropriate to minimize disagreements to the point of dishonesty; they should be dealt with by logical rebuttal in the cover letter and, sometimes, by acknowledging and discussing the point in the manuscript.

Perhaps the most difficult case occurs when you feel that a reviewer shows a bias towards a theoretical approach that differs from yours, and therefore undervalues the work. Here you can explain in the cover letter that there are different approaches to the problem (state what these are), that yours is equally valid, that there is a genuine difference of opinion and that you have a different but scientifically legitimate point of view. However, this strategy is probably unwise unless you have a strong case and there is no other way to deal with the issue. In the end, the editor will have to decide and what one person perceives as objective and unbiased looks very different from another viewpoint. At the end of the day, the editor wants to have articles to publish. The number of acceptances rather than of rejections is therefore the mark of success and of an editor's job well done. Authors, editors, reviewers, and publishers must all work together to ensure the production of a journal of high quality that achieves its intended objectives.

\section{Acknowledgements}

We thank Klaus Mäkelä and Kerstin Stenius for assistance with drafting the material about qualitative research.

Please visit the website of the International Society of Addiction Journal Editors (ISAJE) at www.isaje.net to access supplementary materials related to this chapter. Materials include additional reading, exercises, examples, PowerPoint presentations, videos, and e-learning lessons. 


\section{Appendix A: General Publications on Scientific and Medical Publishing}

This is a very short selection from the huge number of publications. Many additional works may be found by searching biomedical databases such as PubMed or on-line booksellers.

Albert, T. (2000). The A-Z of Medical Writing. London, England: BMJ Books. American Psychological Association. (2010). Publication Manual of the American Psychological Association (6th ed). Washington, DC: Author.

British Medical Association. http://bma.org.uk/about-the-bma/bma-library/ library-guide/reference-styles This is a useful website established by the British Medical Association, giving general guidance on resources for people publishing in the biomedical field.

Hofmann, A. K. (2013). Scientific Writing and Communication: Papers, Proposals, and Presentations. New York, NY: Oxford University Press.

Huth, E. J. (1990). How to Write and Publish Papers in the Medical Sciences (2nd ed). New York, NY: Williams \& Wilkins.

Iverson, C. (Ed.). (1998). American Medical Association Manual of Style: A Guide for Authors and Editors (9th ed.). New York, NY: Williams \& Wilkins.

Katz, M. J. (2009). From Research to Manuscript: A Guide to Scientific Writing (2nd ed.). New York, NY: Springer.

McInerney, D. M. (2002). Publishing Your Psychology Research: A Guide to Writing for Journals in Psychology and Related Fields. Thousand Oaks, CA: Sage.

Moher, D., Schulz, K. F., Altman, D. G., \& for the CONSORT Group. (2001). The CONSORT statement: Revised recommendations for improving the quality of reports of parallel-group randomised trials. The Lancet, 357, 1191-1194.

Peat, J., Elliott, E., Baur, L., \& Keena, V. (2002). Scientific Writing: Easy When You Know How. London, England: BMJ Books.

Richardson, P. (Ed.). (2002). A Guide to Medical Publishing and Writing: Your Questions Answered. London, England: Quay Books.

Strunk, W., Jr., \& White, E. B. (1999). The Elements of Style (4th ed.). New York, NY: Longman.

\section{Appendix B: Checklist of Items to Include When Reporting a Randomized Trial}

This section consists of a slightly shortened version of the checklist from Moher et al. (2001).

TITLE AND ABSTRACT How participants were allocated to interventions (e.g., "random allocation," "randomized," or "randomly assigned"). 
INTRODUCTION

\section{METHODS}

Participants

Interventions

Objectives

Outcomes

Sample size

Randomization

Allocation concealment

Implementation

Blinding

Statistical analysis

\section{RESULTS}

Participant flow:

Recruitment:
Scientific background and explanation of rationale.

Eligibility criteria for participants and the settings and locations where the data were collected.

Precise details of the interventions intended for each group and how and when they were actually administered.

Subjective objectives and hypotheses.

Clearly defined primary and secondary outcome measures and, when applicable, any methods used to enhance the quality of measurements (e.g., multiple observations, training of assessors).

How sample size was determined and, when applicable, explanation of any interim analyses and stopping rules.

Method used to generate the random allocation sequence, including details of any restriction (e.g., blocking, stratification).

Method used to implement the random allocation sequence (e.g., numbered containers or central telephone), clarifying whether the sequence was concealed until interventions were assigned.

Who generated the allocation sequence, who enrolled participants, and who assigned participants to their groups.

Whether participants, those administering the interventions, and those assessing the outcomes were aware of group assignment.

Statistical methods used to compare groups for primary outcome; methods for additional analyses, such as subgroup analyses and adjusted analyses.

Flow of participants through each stage (a diagram is strongly recommended). Specifically, for each group, report the numbers of participants who were randomly assigned, received intended treatment, completed the study protocol, and were analyzed for the primary outcome. Describe protocol deviations from study as planned, together with reasons.

Dates defining the periods of recruitment and follow-up. 
Baseline data:

Numbers analyzed:

Ancillary analyses:

Adverse events:

\section{DISCUSSION}

Interpretation

Generalizability

Overall evidence

Baseline demographic and clinical characteristics of each group.

Number of participants (denominator) in each group included in each analysis and whether the analysis was by "intention to treat." State the results in absolute numbers when feasible (e.g., $10 / 20$, not $50 \%$ ).

Outcomes and estimation: For each primary and secondary outcome, a summary of results for each group, and the estimated effect size and its precision (e.g., 95\%confidence interval).

Address multiplicity by reporting any other analyses performed, including subgroup analyses and adjusted analyses, indicating those prespecified and those exploratory.

All important adverse events or side effects in each intervention group.

Interpretation of the results, taking into account study hypotheses, sources of potential bias or imprecision, and the dangers associated with multiplicity of analyses and outcomes.

\section{External validity of the trial findings.}

General interpretation of the results in the context of current evidence.

Source: Moher, D., Schulz, K. F., \& Altman, D.G., for the CONSORT Group. (2001). The CONSORT statement: Revised recommendations for improving the quality of reports of parallel-group randomised trials. The Lancet, 357, 1191-1194. 